\title{
Iterative refinement and smoothing of cyclone tracks from mean sea level pressure data
}

\author{
D. H. Smith ${ }^{1} \quad$ A. Sulaiman ${ }^{2}$
}

(Received 21 November 2012; revised 8 August 2013)

\begin{abstract}
Local nonlinear regression fits are applied to mean sea level pressure data from 24 member ensemble forecasts of three recent tropical cyclones in the Australian region. Cyclone track estimates derived from the fits are subjected to verification calculations in the form of error-spread diagrams, demonstrating consistency with a recent global ensemble study, with attention also given to cross and along track error components.
\end{abstract}

Subject class: $65 \mathrm{~K} 99$

Keywords: Gauss-Newton iteration

http://journal.austms.org.au/ojs/index.php/ANZIAMJ/article/view/6421 gives this article, (C) Austral. Mathematical Soc. 2013. Published September 13, 2013, as part of the Proceedings of the 16th Biennial Computational Techniques and Applications Conference. ISSN 1446-8735. (Print two pages per sheet of paper.) Copies of this article must not be made otherwise available on the internet; instead link directly to this URL for this article. 


\section{Contents}

1 Introduction

C541

2 Pressure fitting surfaces

C542

2.1 Regression functions . . . . . . . . . . . . . . C543

2.2 Gauss-Newton iteration . . . . . . . . . . . . . C544

2.3 Sensitivity of fit parameters to data . . . . . . . . C545

3 Numerical results

C545

3.1 Tracks and parameter sensitivity . . . . . . . . C C547

3.2 Error-spread diagrams . . . . . . . . . . . . . C550

3.3 Cross track and along track errors . . . . . . . . . C552

4 Summary and conclusions

C554

References

C554

\section{Introduction}

Vorticity, circulation and mean sea level pressure (MSLP) are important quantities in the identification and characterisation of tropical cyclones [8]. For tracking purposes, one class of methods estimates cyclone pressure centres from forecast or observed data by parametric model fitting, including sinusoidal functions along zonal and meridional directions [1], exponential inverse powers of distance [4, 5], and ellipsoids in polar stereographic coordinates [6]. A unique local minimum of the model function then provides the cyclone track position and central pressure estimate.

This study explores the application of two regression functions, each requiring fewer than ten parameters, to archived MSLP ensemble forecast data for three recent tropical cyclones in the Australian region, as generated by the ACCESS global and regional ensemble prediction system, AGREPS [7]. With a spatial 
resolution of $0.375^{\circ}$, or approximately $40 \mathrm{~km}$, off-grid pressure centre estimates are necessary for accuracy and smoothness considerations [6]. Parameter estimation is achieved using Gauss-Newton iteration [2], with associated sensitivity calculations also carried out to assess dependence on the forecast MSLP data. The resulting cyclone track estimates are subjected to further analysis in the form of error-spread diagrams, allowing comparison with some published global ensemble results [3], and the calculation of cross and along track error components [9].

\section{Pressure fitting surfaces}

Near the centre of a tropical cyclone, mean sea level pressure is locally approximated by an appropriate 'depression function' [4, 6]

$$
p(\theta, \phi) \approx f(\theta, \phi ; \mathbf{q})
$$

where $\theta$ is longitude, $\phi$ is latitude, and $\mathbf{q}$ contains parameters to be estimated from MSLP forecast data. For AGREPS regional forecasts [7], the data comprises 24 ensemble members, each starting from slightly different initial conditions, together with the ensemble mean and associated verifying analysis, every six hours up to $T+72$ hours where $T$ represents the forecast start time. Tropical cyclone (TC) Yasi crossed the Queensland coast during the early hours of February 3, 2011. Most of its lifespan was captured by the 72 hour ensemble forecast started at 18 UTC on January 30, 2011, with landfall occurring a few hours before $T+72$. About two weeks later, TC Dianne and TC Carlos were simultaneously active, Dianne off the West Australian coast, and Carlos in the Darwin area, both captured by an ensemble forecast started at 06 UTC on February 16, 2011. 


\subsection{Regression functions}

A version of Holland's exponential funnel shaped fitting function for pressure $p$ [4], characterised by a pressure minimum $p_{0}$, depression depth $a$, and radius parameter $b$, is

$$
p=p_{0}+a \exp \left[\frac{-b}{\left|s(\theta, \phi)-s\left(\theta_{0}, \phi_{0}\right)\right|^{2}}\right],
$$

where $\left(\theta_{0}, \phi_{0}\right)$ is the estimated cyclone centre, and $s(\theta, \phi)$ represents spherical to cartesian coordinate conversion. The fit (1) has wide application, and may be extended to allow cyclone translation during core pressure observations during the measurement period [5], but may suffer issues in the presence of asymmetry.

Murray and Simmonds catered for asymmetry with an ellipsoid of best fit, iteratively generated using spatial derivatives provided from bicubic spline interpolation on a polar stereographic grid [6], selected for its near conformal properties. Retaining the polar stereographic grid of Murray and Simmonds [6], and employing an elliptic paraboloid

$$
p=p_{0}+\frac{\left[\left(x-x_{0}\right) \cos \alpha+\left(y-y_{0}\right) \sin \alpha\right]^{2}}{a^{2}}+\frac{\left[\left(y-y_{0}\right) \cos \alpha-\left(x-x_{0}\right) \sin \alpha\right]^{2}}{b^{2}},
$$

also accommodates asymmetry, with less restrictions on the fit geometry parameters than the ellipsoid case. The pressure minimum is $p_{0}$, geometrical parameters $a$ and $b$ control the asymmetry, $\alpha$ is a rotation angle in polar stereographic space, and the centre is at $\left(x_{0}, y_{0}\right)$. Transforming from longitudelatitude coordinates $(\theta, \phi)$ to polar stereographic coordinates $(x, y)$,

$$
x=\cos \phi \cos \theta, \quad y=\cos \phi \sin \theta .
$$

Estimating the five parameters for (1), or six parameters for (2), now proceeds by defining an appropriate set of data points, centred on the local grid minimum, and applying Gauss-Newton iteration [2]. For the ensemble forecasts 
under consideration, this process is applied for each of the 24 members, the ensemble mean, and verifying analysis, every six hours.

\subsection{Gauss-Newton iteration}

The residual $\boldsymbol{r}_{j}$ for grid point $j$ at $\left(\theta_{j}, \phi_{j}\right)$ is the difference between the grid pressure value $p_{j}$ and its approximate fit $f$,

$$
r_{j}\left(\mathbf{q} ; p_{j}\right)=f\left(\theta_{j}, \phi_{j} ; \mathbf{q}\right)-p_{j} .
$$

The cost function $\mathbf{c}\left(\mathbf{q} ; \mathfrak{p}_{\mathbf{j}}\right)$ to be minimised is half the sum of squared residuals

$$
c\left(\mathbf{q} ; p_{j}\right)=\frac{1}{2} \mathbf{r}^{\top} \mathbf{r}
$$

and has a gradient with respect to the fit parameters $\mathbf{q}$ of

$$
\nabla c\left(\mathbf{q} ; p_{j}\right)=\mathbf{g}\left(\mathbf{q} ; p_{j}\right)=\sum_{j} r_{j} \nabla r_{j}=A\left(\mathbf{q} ; p_{j}\right)^{\top} \mathbf{r} .
$$

Matrix $A\left(\mathbf{q} ; p_{j}\right)$ is the Jacobian of the residual function, and second derivatives of $\mathbf{c}\left(\mathbf{q} ; p_{j}\right)$ are

$$
\nabla^{2} \mathrm{c}\left(\mathbf{q} ; p_{j}\right)=A^{\top} A+\sum_{j} r_{j} \nabla^{2} r_{j}
$$

Gauss-Newton iteration [2] applies quasi-Newton iteration to zero the gradient function, or pursue stationary points of the cost function $c\left(q ; p_{j}\right)$, with Hessian approximation provided by first derivatives only. Iteration $k$ proceeds by solving a linear system for the parameter increments $\boldsymbol{\delta}_{\mathrm{k}}$, and then updating the parameters according to

$$
A_{k}^{\top} A_{k} \delta_{k}=-A_{k}^{\top} r_{k}, \quad \mathbf{q}_{k+1}=\mathbf{q}_{k}+\boldsymbol{\delta}_{k},
$$

until convergence is achieved. 


\subsection{Sensitivity of fit parameters to data}

For a converged solution,

$$
\mathbf{g}\left(\mathbf{q} ; p_{j}\right)=0
$$

differentiating with respect to the grid pressure data $p_{j}$ gives

$$
\frac{\partial g}{\partial \mathbf{q}} \frac{\partial \mathbf{q}}{\partial p_{j}}+\frac{\partial g}{\partial p_{j}}=0
$$

which constitutes a linear system of equations for the sensitivities $\partial \mathbf{q} / \partial p_{j}$. Unlike the Gauss-Newton iterations, which provide the initial solution $\mathbf{q}$, an exact second derivative matrix is used, as provided by the expression (3).

\section{$3 \quad$ Numerical results}

For a 72 hour ensemble forecast, cyclone tracks were estimated for each ensemble member, the ensemble mean, and the associated verifying analysis, every six hours. Best results were achieved via an 'outer loop' in members and 'inner loop' in time strategy, whereby all parameter vectors for a given ensemble member are calculated sequentially for its entire forecast, with initial pressure centre guess provided by the gridpoint minimum, and remaining geometrical parameter guesses taken from the previous time step. With this approach the elliptic paraboloid fit (2) demonstrated superior robustness by rapidly converging in 5-10 iterations for all members and all times, with an applied exit tolerance of $10^{-7}$ on the difference norm between successive iterates, $\sqrt{\left(\mathbf{q}_{k+1}-\mathbf{q}_{k}\right)^{\top}\left(\mathbf{q}_{k+1}-\mathbf{q}_{k}\right)}$, and final gradient norms in the vicinity of $10^{-9}$. The axisymmetric fit (1) occasionally failed to converge, but recovered after some adjustment of the initial guess, typically needing 10-20 iterations and yielding a final gradient in the vicinity of $10^{-4}$.

A promising alternative iteration strategy under consideration eliminates the inner time loop, and updates entire members, often proving effective at recovering individual members which fail at occasional times. This naturally leads 


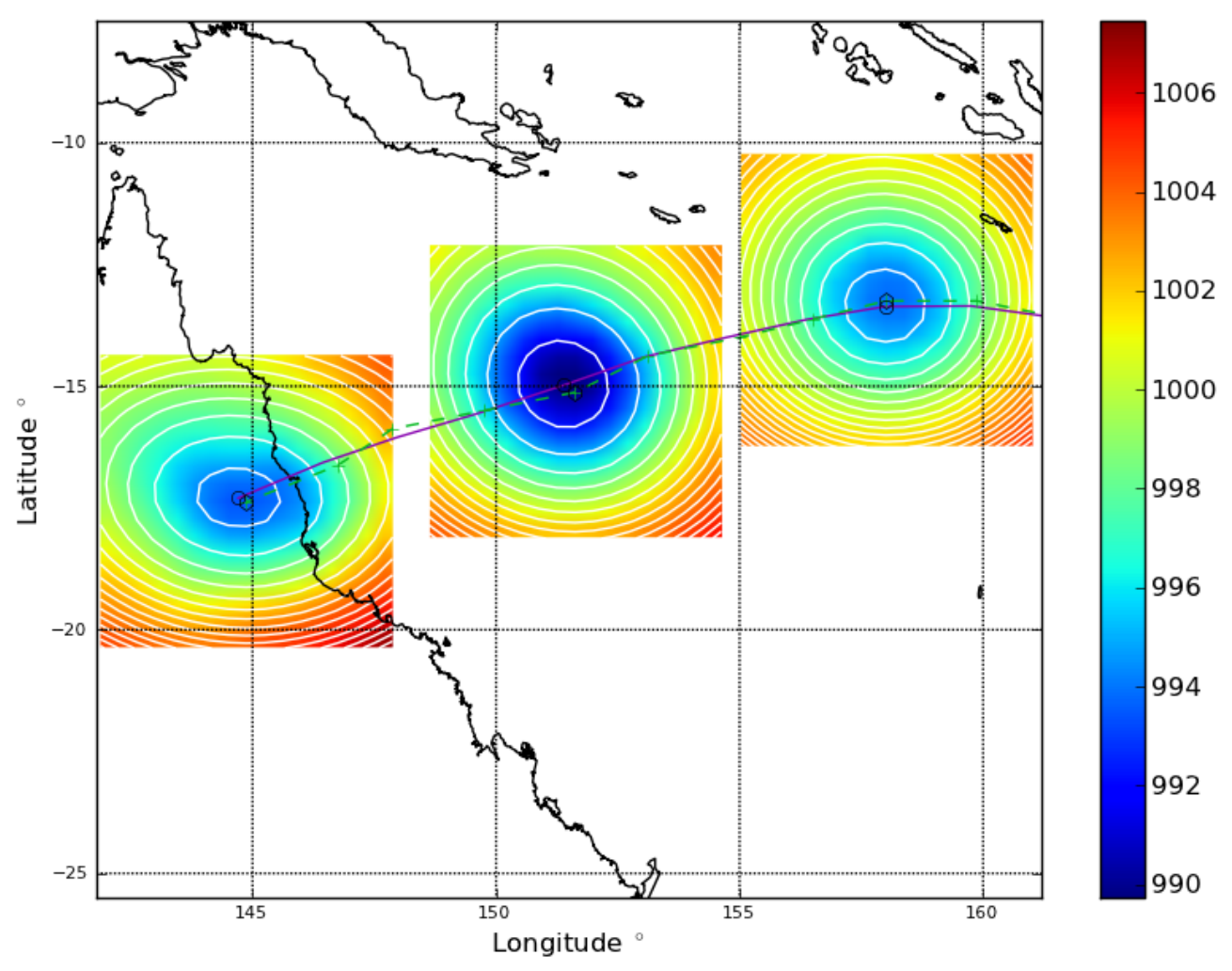

Figure 1: Elliptic paraboloid fit results for the ensemble mean forecast of TC Yasi, showing fit as white contours with background colours describing the MSLP forecast data in $h P a$, at times $\mathrm{T}+24, \mathrm{~T}+48$ and $\mathrm{T}+72$, moving right to left. Fitting was performed on a $11 \times 11$ grid centred on the grid minimum, with the minimum shown as a broken green line, and evaluated on an extended $17 \times 17$ area for visualisation. Pronounced asymmetry is evident after landfall at $\mathrm{T}+72$. 
to the possibility of also eliminating the member loop, thus simultaneously updating all members and times, with 'super' parameter vectors containing the entire ensemble forecast.

\subsection{Tracks and parameter sensitivity}

For TC Yasi, Figure 1 gives the ensemble mean track estimate produced by elliptic paraboloid fitting on an $11 \times 11$ submesh centred on the grid minimum, also marked as a broken line, with detailed snapshots at $\mathrm{T}+24,48$ and 72 . At each of these times the fit was evaluated on an extended $17 \times 17$ region, centred on the grid minimum, with white contours indicating the fit geometry and background colour shading showing the actual forecast data. Of the three snapshots shown, $\mathrm{T}+48$ is the most intense, at about $990 \mathrm{hPa}$, and also displays the highest degree of symmetry. Upon landfall, the elliptical contours, strictly valid only in polar stereographic space, elongate in the east-west direction to give a pronounced asymmetry.

For the track estimate of Figure 1, departures from the associated grid minima were all below the grid spacing of $0.375^{\circ}$, except for a longitude displacement of just below 1.2 grid spacings at $T+0$ when the depression was relatively shallow and broad. A similar situation occurred for ensemble member 14 which produced the largest deviation of just over seven grid intervals in latitude, positioning the track estimate outside the fitting points. Graphical inspection of this result revealed the grid minimum to be a local 'spot' near the edge of a large depressed region, which was effectively treated as part of an elliptical area by the Gauss-Newton algorithm, producing a visually acceptable picture.

Sensitivity results for Figure 1, showing partial derivatives of the fit minimum pressure $p_{0}$ with respect to the grid data used in the fit calculation (4), appear in Figure 2 with the associated fit contours. Each case is characterised by a local positive maximum around the track position estimate, where reductions in grid pressure act to decrease the fitted minimum $p_{0}$, indicating favourable 


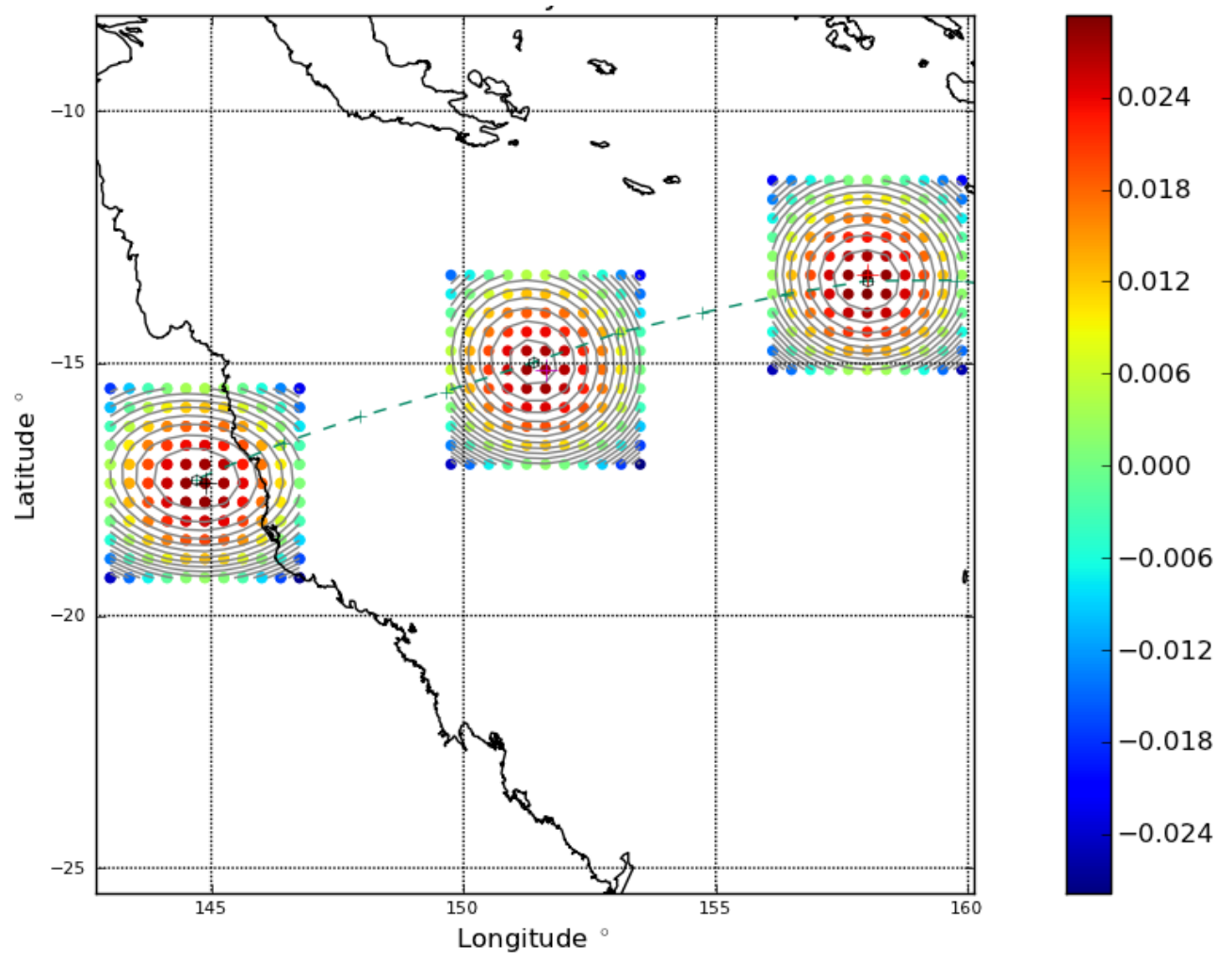

Figure 2: Sensitivity of fit minimum pressure $p_{0}$ to grid pressure data for the results shown in Figure 1, including the fit contours limited to the $11 \times$ 11 fitting points. A positive neighbourhood around the estimated track position indicates 'favourable' fitting points which act to reduce $p_{0}$. 


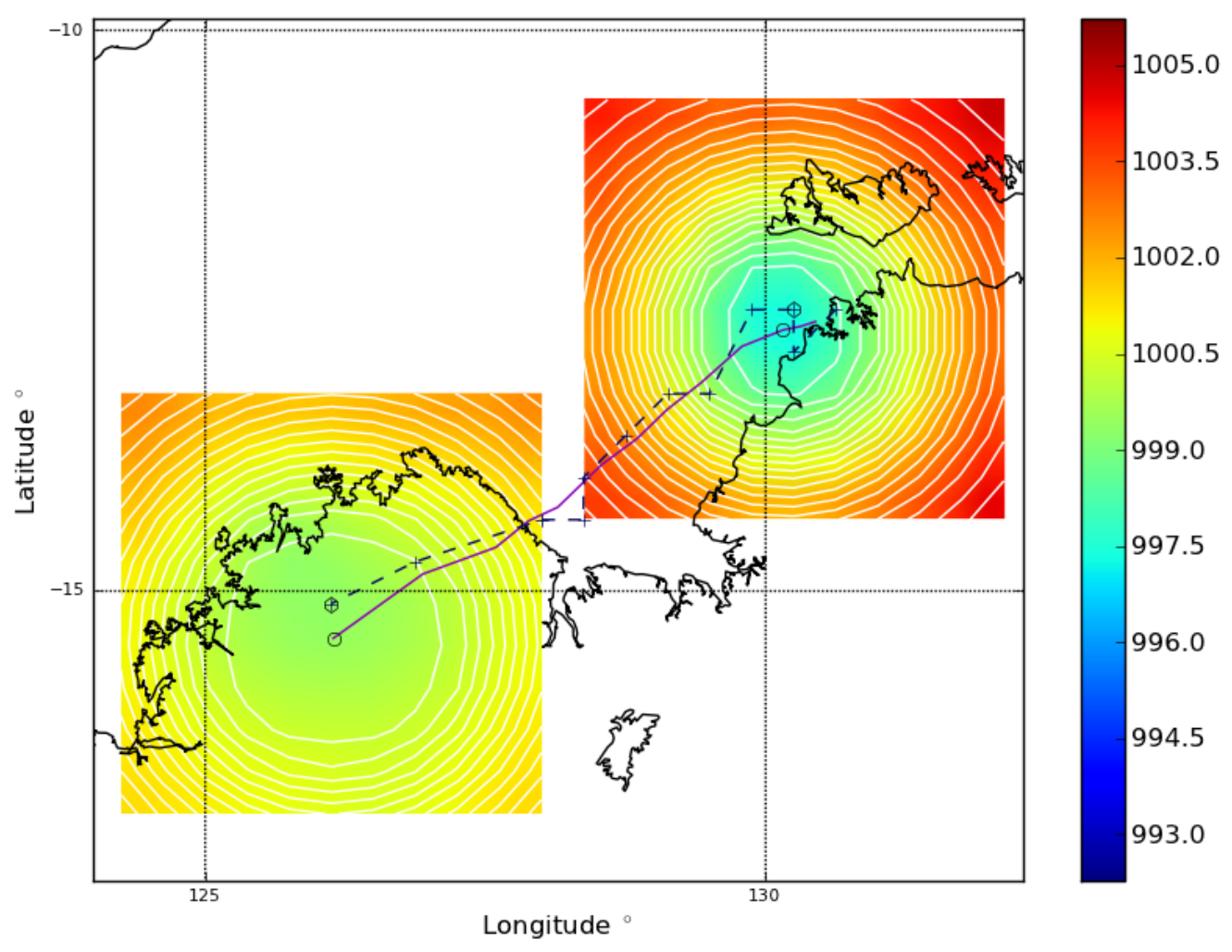

Figure 3: Axisymmetric fit results (1) for the ensemble mean forecast of TC Carlos at $T+12$ and $T+72$, moving from upper right to lower left, showing white fit contours against background colours of the MSLP forecast data in $\mathrm{hPa}$. Fitting and evaluation was performed on an $11 \times 11$ grid centred on the grid minimum (broken line). 
fitting points. As the fit contours expand away from the track the sensitivity weakens and changes sign, revealing unfavourable fitting points that cut the corners off the original fitting square. Regarding the actual values of $p_{0}$, these were usually slightly above the grid minima, up to $1.5 \%$ for the ensemble mean track estimate of Figure 1.

For the axisymmetric fit (1), Figure 3 shows an ensemble mean track estimate (TC Carlos), together with grid minima, forecast data and detailed snapshots at $T+12$ and $T+72$, on the original $11 \times 11$ fitting set. In this diagram the smoothing effect of the fitting process is particularly evident on comparison of the grid minima with the estimated track, with departures slightly exceeding the grid spacing on three occasions.

The fitting results of Figures 1 to 3 constitute a tiny sample of those available for the three ensemble forecasts, which will now be summarised by considering track error and spread metrics, with a brief consideration of along and cross track error components.

\section{$3.2 \quad$ Error-spread diagrams}

Spread-error consistency is a key performance indicator for ensemble forecasts, receiving particular attention in a comprehensive study of tropical cyclone forecasts during 2009, involving six global ensembles from major world centres [3]. In this study, Hamill et al. [3] constructed cyclone track error-spread diagrams with error defined as the great circle deviation of the ensemble mean's track from the verifying position, and spread defined as the average separation distance of the ensemble member tracks from that of the ensemble mean, without specifying actual tracking methods. Hamill et al. [3] concluded that there is an "urgent need" for improved tropical cyclone track and intensity forecasts.

Using the error and spread measures of Hamill et al. [3], with track estimates generated from the elliptic paraboloid fit (2), an error-spread diagram for 


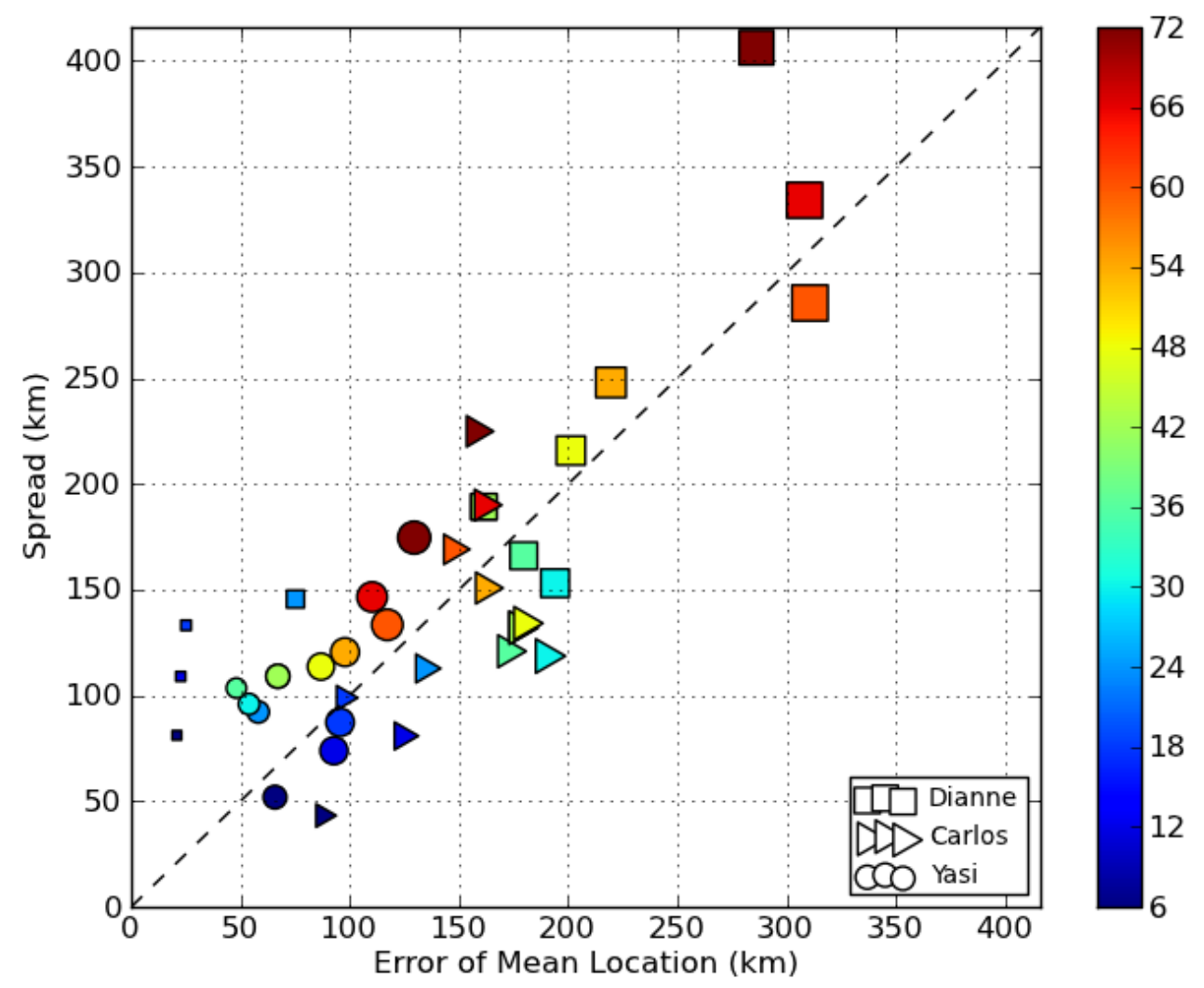

Figure 4: Error-spread scatter plots for all three cyclone forecasts, using estimated track data from the elliptic paraboloid fit (2), on an $11 \times 11$ fitting area. Results are given every six hours, with colour by time and size by error. 
the three tropical cyclones under consideration is given in Figure 4, showing results every six hours. As noted by Hamill et al. [3], calibrated ensemble systems should provide consistency between error and spread, or populate the diagonal vicinity. In this respect, overspreading is evident for most of the TC Yasi forecast, whereas TC Carlos is mostly underspread, and TC Dianne shows early underspreading and crosses the diagonal later in the forecast. Overall, the picture displays a reasonable population balance between each side of the diagonal.

The largest error-spread box in Figure 4 is for the TC Dianne forecast, with a spread of just over $400 \mathrm{~km}$, followed by TC Carlos at approximately $230 \mathrm{~km}$, then TC Yasi at $180 \mathrm{~km}$. These fit well inside the $800 \mathrm{~km}$ boxes of Hamill et al. [3], which were constructed from many more storm forecasts (between 25 and 114), offering a useful check on the AGREPS ensemble's performance. An additional check involves repeating the error-spread calculations of Figure 4 with grid minima replacing the track estimates. This check indicates maximum displacements in error of less than $50 \mathrm{~km}$ for TC Yasi and TC Carlos, and for TC Dianne, within $100 \mathrm{~km}$. Maximum associated spread deviations over all three cases reached just over $30 \mathrm{~km}$.

\subsection{Cross track and along track errors}

Following the methodology outlined by Heming [9], along and cross track error components were calculated for the track estimates. At any forecast time, the current best track position and its predecessor, six hours earlier, are connected by a great circle arc which intersects a second great circle arc passing through the current estimated track position at $90^{\circ}$. Connecting this intersection point, obtained by solving a pair of nonlinear equations, to the current best track point gives the along track component, and connection with the current estimated track point constitutes the cross track component.

For TC Dianne, which occupied the largest error-spread box in Figure 4, ensemble member 12 crossed the coast and finished $1465 \mathrm{~km}$ from the best 


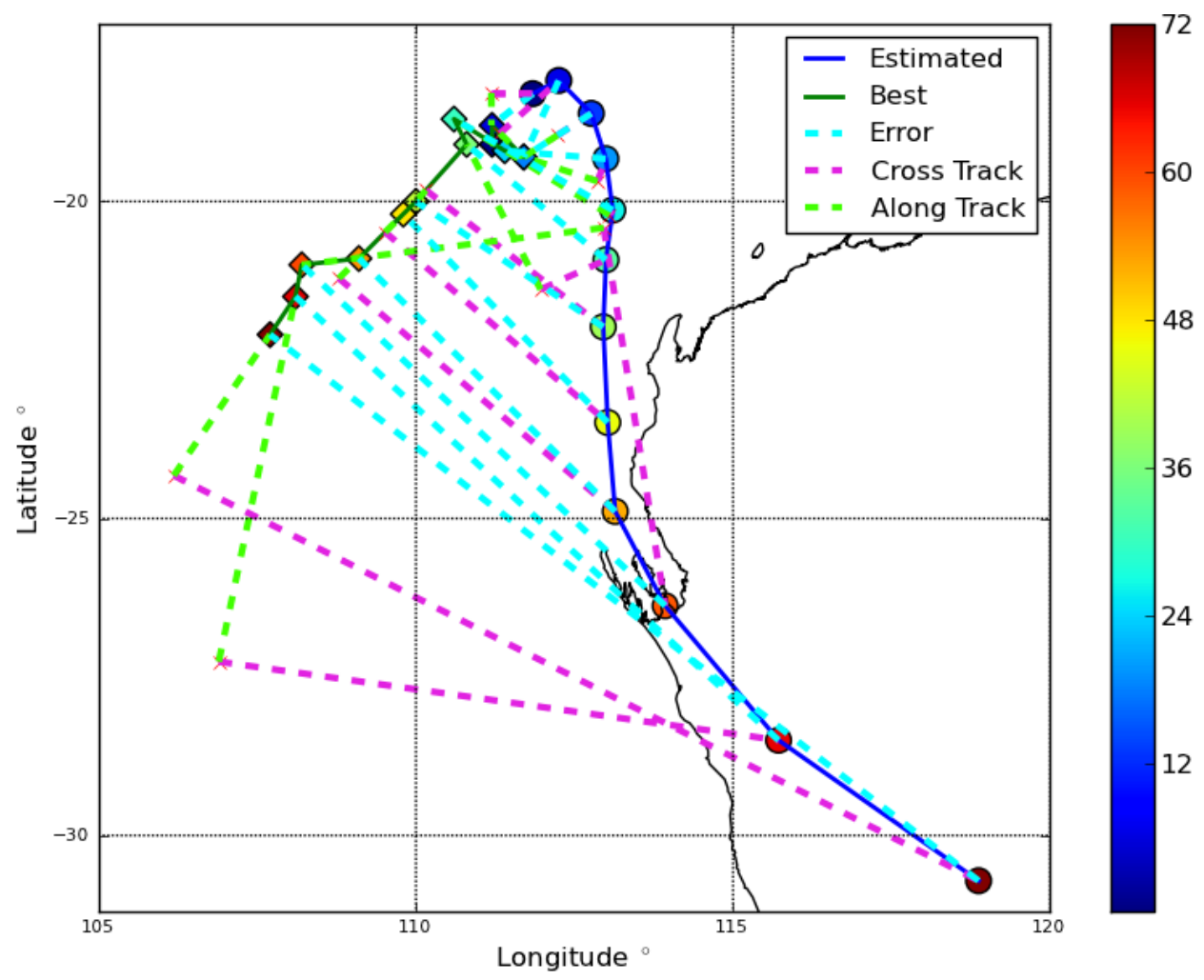

Figure 5: Track error behaviour, including along and cross components, for ensemble member 12 of the TC Dianne forecast, with track estimate provided by elliptic paraboloid fitting (2) on an $11 \times 11$ fitting area, and markers coloured by time. At $T+72$ the deviation from the best track was $1465 \mathrm{~km}$. 
track at $T+72$. Along and cross track error components for this member, calculated from the elliptic paraboloid track estimate (2), are shown in Figure 5, indicating a strong cross track contribution commensurate with the total error for most of the forecast.

\section{Summary and conclusions}

Two nonlinear regression fits, one symmetric and one allowing asymmetry, were applied to archived three day regional ensemble forecast MSLP data for three recent tropical cyclones in the Australian region, with parameter estimation by Gauss-Newton iteration. The asymmetric case, an elliptic paraboloid in polar stereographic coordinates, demonstrated superior robustness and confirmed a need for pressure asymmetry on multiple occasions. Subsequent track error and spread calculations for the three cyclones placed the ensemble results well within the error-spread boxes of a recent comprehensive global ensemble study. However, more storms need to be considered for a fairer comparison. Future studies will address this deficiency, in addition to asymmetry, parameter sensitivity, and meaningful ensemble measures involving total, along track, and cross track error components.

Acknowledgements The authors are grateful for constructive comments provided by two anonymous referees.

\section{References}

[1] R. E. Benestad and D. Chen, The use of a calculus-based cyclone identification method for generating storm statistics, Tellus A 58(4):473-486 (2006). doi:10.1111/j.1600-0870.2006.00191.x C541 
[2] R. Fletcher, Practical Methods of Optimization, Wiley (1987). C542, C543, C544

[3] T. M. Hamill, J. S. Whitaker, M. Fiorino and S. G. Benjamin, Global Ensemble Predictions of 2009's Tropical Cyclones Initialized with an Ensemble Kalman Filter, Mon. Wea. Rev. 139:668-688 (2011). doi:10.1175/2010MWR3456.1 C542, C550, C552

[4] G. J. Holland, An Analytic Model of the Wind and Pressure Profiles in Hurricanes, Mon. Wea. Rev. 108:1212-1218 (1980). doi:10.1175/1520-0493(1980)108<1212:AAMOTW>2.0.CO;2 C541, C542, C543

[5] J. D. Kepert, Objective Analysis of Tropical Cyclone Location and Motion from High-Density Observations, Mon. Wea. Rev. 133:2406-2421 (2005). doi:10.1175/MWR2980.1 C541, C543

[6] R. J. Murray and I. Simmonds, A numerical scheme for tracking cyclone centres from digital data Part I: development and operation of the scheme, Australian Meteorological Magazine 39:155-166 (1991). http://www.bom.gov.au/amoj/docs/1991/murray1.pdf C541, C542, C543

[7] D. H. Smith, M. Naughton and A. Sulaiman, Multiscale verification calculations for regional ensemble forecasts, ANZIAM J. 52:C882-C898 (2011). http://journal austms.org.au/ojs/index.php/ANZIAMJ/ article/view/3936 C541, C542

[8] M. R. Sinclair, Objective Identification of Cyclones and Their Circulation Intensity, and Climatology, Weather and Forecasting 12:595-612 (1997). doi:10.1175/1520-0434(1997)012<0595:OIOCAT>2.0.CO;2 C541

[9] J. Heming, Tropical cyclone forecast verification method, UK Met Office, (2012).

http://www.metoffice.gov.uk/weather/tropicalcyclone/method 
See also doi:10.1175/1520-0493(1998)126<1323:TPOTUK > 2.0.CO;2 C542, C552

\section{Author addresses}

1. D. H. Smith, Center for Australian Weather and Climate Research, Bureau of Meteorology, Melbourne Australia mailto:dhsmith@bom.gov.au

2. A. Sulaiman, Center for Australian Weather and Climate Research, Bureau of Meteorology, Melbourne Australia 